\title{
Experimental evidence for multi-pass extraction with a bent crystal
}

\author{
B. Dehning, K. Elsener, G. Fidecaro, M. Gyr, W. Herr, J. Klem, W. Scandale, G. Vuagnin, \\ E. Weisse, S. Weisz (CERN); S.P. Møller, E. Uggerhoj (Aarhus University, Denmark); \\ A. Freund, R. Hustache (ESRF Grenoble); G. Carboni (Roma, Italy); \\ M.P. Bussa, F. Tosello (Torino, Italy)
}

\section{ABSTRACT}

The feasibility of extracting particles from the halo of a circulating proton beam using a bent silicon crystal has been demonstrated experimentally at the SPS for a beam energy of 120 $\mathrm{GeV}$. Presently studies are conducted to understand the extraction mechanisms and the measured efficiencies. In particular the contribution of multi-pass extraction, where the particles can pass through the crystal many times before being channeled and extracted, is investigated. In a recent experiment, using a crystal especially fabricated with a finite amorphous layer on its surface, it has been proven that multi-pass extraction plays an important role. The experiment is described and the implication for further studies are discussed.

\section{INTRODUCTION}

There is considerable theoretical and experimental activity in order to understand the mechanisms leading to the observed proton beam intensities extracted by means of a bent crystal from high energy accelerators $[1,2,3,4]$. One aim of this work is to assess the perspectives of using such a crystal to parasitically extract a proton beam from the LHC [5].

While the principles of channeling and deflection of protons in bent silicon crystals are well understood [6], the specific processes leading to the extraction of protons from the halo of a circulating beam are more difficult to assess. The extraction of 120 $\mathrm{GeV}$ protons from a beam coasting in the CERN SPS is now regularly achieved with an efficiency of about 10\% [3]. Two mechanisms are being discussed, which could be responsible for extraction with a bent crystal: first-pass extraction and multi-pass extraction. The aim of the experiment described in this report was to experimentally demonstrate the existence of the latter.

\section{EXPERIMENT AT THE SPS}

For the present experiment a $120 \mathrm{GeV}$ coasting beam with an intensity of about $5 \cdot 10^{11}$ protons was used. The lifetime of the beam without external interference can be several hundred hours. The crystal is placed typically at 10 from the closed orbit, some 10 times the transverse beam size, where only very few halo particles are found initially. The beam is then excited horizontally with band limited white noise induced on a pair of condenser plates. Typical kicks produced are of order $0.0005-0.005$ $\mu \mathrm{rad}$, and the kick strength can be varied by changing the noise amplitudes. Once the beam has diffused onto the crystal and a steady state is reached, normalized emittances of $60 \mathrm{~mm} \mathrm{mrad}$ (horizontal) and $10 \mathrm{~mm} \mathrm{mrad}$ (vertical, unaffected by the noise) are found. Beam lifetimes in this steady state vary from a few minutes to 50 hours depending on the kick strength. The protons extracted by the bent crystal are measured in an arrangement of detectors which is described in refs. $[3,4]$.

\section{FIRST- AND MULTI-PASS EXTRACTION}

First-pass extraction would correspond to the experimental situation found at an external beam-line [6], the only difference being the small impact parameters of the protons onto the crystal (see next section). Therefore, for first-pass extraction the surface quality is of paramount importance.

Multi-pass extraction has been predicted as a possible mechanism by several authors [7, 8]. The term "multi-pass" refers to the situation of protons that are not channeled during their first encounter with the crystal, but rather scattered by a small angle. Thus these protons will have "further chances" at later turns to be channeled and extracted when passing the crystal inside the critical angle for channeling (see [3]) and with larger impact parameters. While for a first-pass extraction with the required high efficiency the surface properties of the crystal are important, the accelerator parameters play a more important role for the multipass mechanism [9].

No direct experimental proof of the existence of this mechanism existed so far, although some (model-dependent) conclusions were inferred from recent experimental results. For example, the observation of extracted protons over a very large range of crystal angles with respect to the closed orbit in the SPS experiment [3], but also the enhanced extraction efficiency due to an additional scatterer observed at Serpukhov [10] seem to indicate the importance of multi-pass extraction.

Within the restrictions of an accelerator experiment, the possibility to reach large impact parameters (therefore clear "firstpass" conditions) are limited: the only possibility found is the "kick-mode" [11], but the high instantaneous proton intensities extracted in this mode make it hard to estimate an extraction efficiency. Furthermore, such a mode does not reflect the standard operational conditions and certainly cannot be used for a parasitic extraction in the LHC. Therefore, a different approach was taken here to experimentally prove the importance of multi-pass extraction: a crystal as shown in Fig. 1, with an amorphous layer of $30 \mu \mathrm{m}$ Silicon oxide, has been installed in the SPS [12]. This layer should prevent any first-pass extraction, the impact parameters being much smaller than the thickness of the layer (see below). An extracted beam would therefore demonstrate the existence of multi-pass extraction and provide a clean sample to study the multi-pass process separately and in detail. 


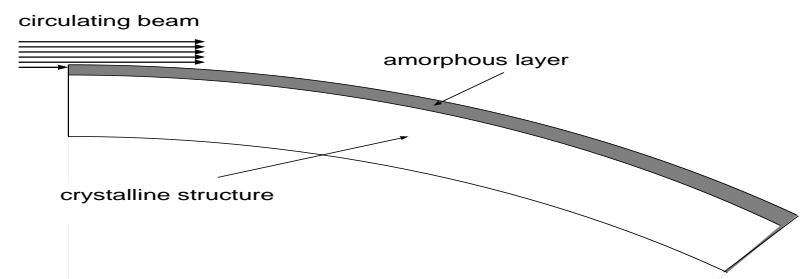

Figure 1: Schematic view of crystal with amorphous layer

\section{COMPUTER SIMULATIONS}

The diffusion of particles in our experiment is provoked by random kicks [13]. The amplitudes of the kicks follow a Gaussian distribution with a width $\sigma_{k}$.

For an otherwise linear machine the diffusion speed is then independent of the betatron amplitude and can reach maximum values in the order of $\mathrm{mm}$ per minute. The magnitude of the impact parameter $b$ can then be estimated by:

$$
b=\frac{D \cdot n_{\text {res }}}{\nu_{\text {rev }}}
$$

where $\mathrm{D}$ is the diffusion speed, $\mathrm{n}_{\text {res }}$ the order of the resonance and $\nu_{\text {rev }}$ the revolution frequency $(\approx 43375 \mathrm{~Hz}$ in the SPS $)$ and gives values in the order or a few $\mathrm{nm}$, in any case much smaller than the crystal imperfections.

In order to get a better understanding of the extraction experiment with a bent crystal at the SPS, particle tracking with a computer program was done $[13,14]$. A beam of $10^{\prime} 000$ particles was followed through the (linear) machine, with parameters close to the ones found in the SPS experiment. Emittance growth due to induced voltage (white noise) on the damper plates was simulated, and the impact parameter and impact angle distributions resulting at the crystal position were recorded. For details of the simulation see $[13,14]$. An example is shown in Fig. 2 for a kick strength of $0.01 \mu \mathrm{rad}$. The impact parameters are very

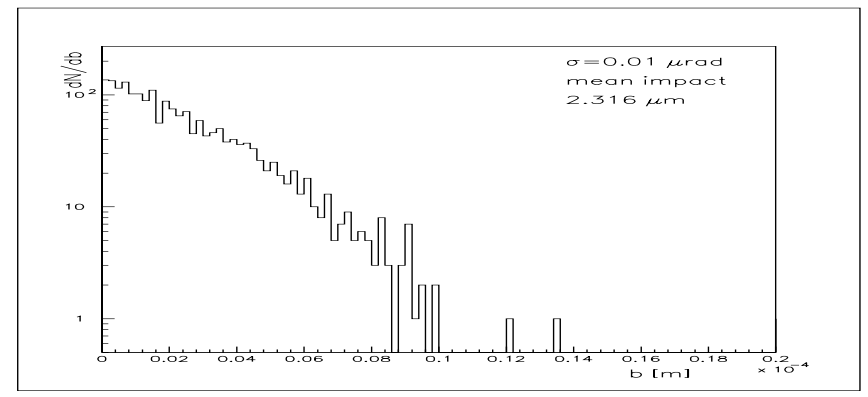

Figure 2: Impact parameter distribution from simulation

small and do not exceed values above $10 \mu$ m even for these kick amplitudes 20 times larger than those used in the experiment. The emittance growth is a stochastic process and very slow when the kicks are small. In order to reach an equilibrium state for many particles, the required time for the simulation is very large and depends strongly on the kick strength. Realistic kick strength would thus require huge amount of computer time. However, as has been shown earlier [13], for small kicks the mean impact parameter rises approximately linearly with the kick strength and scaling of the impact parameter distributions obtained is thus permitted. The important result at this stage is that the mean impact parameter (of an exponential distribution) in the experiment is estimated to be about $20-50 \mathrm{~nm}$ and excludes first-pass extraction.

\section{EXPERIMENTAL RESULTS}

Results of the measurements using the bent silicon crystal with its amorphous layer are summarized in Table 1. Measurements

\begin{tabular}{|c|c|c|c|}
\hline $\begin{array}{c}\text { Intensity } \\
\left(10^{11} \mathrm{p}\right)\end{array}$ & $\begin{array}{c}\text { Lifetime } \\
\text { (hours) }\end{array}$ & $\begin{array}{c}\text { Extracted rate } \\
\left(10^{5} \text { counts/s }\right)\end{array}$ & $\begin{array}{c}\text { Efficiency } \\
(\%)\end{array}$ \\
\hline 5.8 & $33.0 \pm 1.1$ & $2.07 \pm .02$ & $4.2 \pm .3$ \\
\hline 4.5 & $8.1 \pm .2$ & $9.49 \pm .03$ & $6.2 \pm .3$ \\
\hline 4.4 & $28.3 \pm 1.4$ & $2.38 \pm .03$ & $5.5 \pm .5$ \\
\hline 1.9 & $5.11 \pm .2$ & $8.78 \pm .04$ & $8.8 \pm .4$ \\
\hline 1.8 & $2.3 \pm .1$ & $13.83 \pm .12$ & $6.5 \pm .3$ \\
\hline 0.69 & $0.8 \pm .1$ & $13.76 \pm .06$ & $5.9 \pm .3$ \\
\hline 0.57 & $1.7 \pm .1$ & $7.27 \pm .05$ & $8.5 \pm .6$ \\
\hline 0.36 & $0.50 \pm .05$ & $11.06 \pm .35$ & $6.5 \pm .4$ \\
\hline
\end{tabular}

Table 1: Extraction efficiencies for the crystal with an amorphous layer.

were taken for different kick strengths and a crystal position 10 $\mathrm{mm}$ from the closed orbit [15]. The number of particle hitting the crystal is estimated from the beam intensity and the measured lifetime. Extraction efficiencies (defined as the ratio between extracted and lost particles) are rather independent of the kick strength (i.e. impact parameter b) and are in the same order of magnitude as found without amorphous layer [3]. From this result we conclude the existence of multi-pass extraction. The comparable efficiency also indicates that this mechanism plays an important role in the extraction process. The large thickness of the amorphous layer causes proton losses even on later turns, thus reducing the efficiency slightly. A typical angular scan obtained with this crystal is shown in Fig. 3. The width of the scan is approximately $300 \mu \mathrm{rad}$ and the comparison with an angular scan for the same crystal without the amorphous layer shows a similar result [3] and further supports our conclusion that multipass extraction is a dominant mechanism. The profiles of the extracted beam as measured by the scintillator hodoscope at the peak of the angular scan are shown in Figs. 4 and 5. For comparison, measurements were also performed with a so-called "Ushaped" crystal (see [4]), and efficiencies of up to $16 \%$ have been found. Detailed results are published elsewhere [15].

\section{FURTHER STUDIES AND IMPROVEMENTS}

Once the existence and importance of the multi-pass process is established, one can try to increase the extraction efficiency. It has been demonstrated [9] that the efficiency for pure multi-pass extraction strongly depends on the machine parameters, in particular on the $\beta$-function at the crystal position and the tune. 


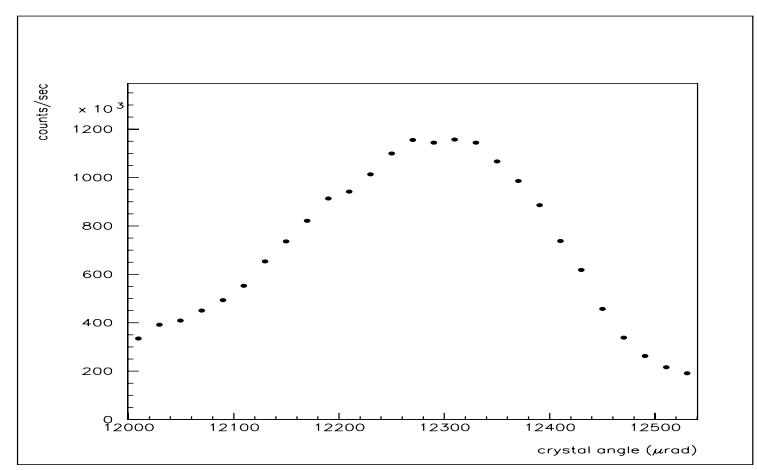

Figure 3: Angular scan with respect to beam axis

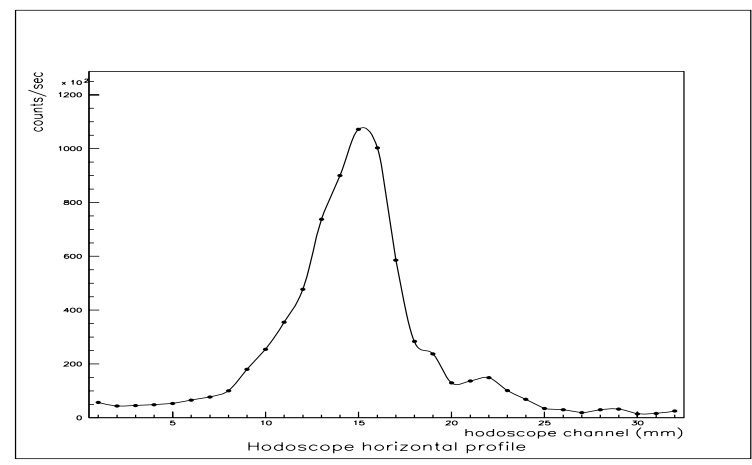

Figure 4: Horizontal profile of extracted beam

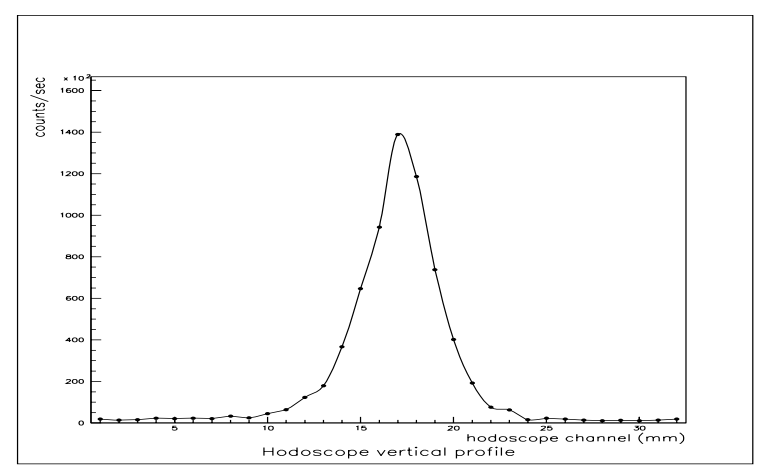

Figure 5: Vertical profile of extracted beam

These parameters can be optimized to achieve the highest possible efficiency [9]. This could be studied with a simulation program or experimentally using a dedicated insertion at the crystal position.

A further possibility has been suggested to increase the efficiency: the use of an external scatterer at an appropriate location in the machine [10]. We intend to evaluate such a scheme in simulations as well as in our experiment at the SPS.

\section{CONCLUSION}

We have investigated the mechanism of multi-pass extraction using a bent crystal with an imperfect surface and have made the following observations:
- We have measured extracted particles using a crystal with an amorphous layer of $30 \mu \mathrm{m}$.

This can only be explained by a multi-pass extraction mechanism.

- The efficiency and angular scans are comparable to the one obtained without an amorphous layer and prove the importance of this mechanism for the extraction process.

\section{REFERENCES}

[1] A. Asseev et al.; Nucl. Instr. Meth. A330 (1993) 39.

[2] R.A. Carrigan et al.; Nucl. Instr. Meth. B90 (1994) 128.

[3] H. Akbari et al.; Phys. Lett. B313 (1993) 491.

[4] H. Akbari et al.; Proc. EPAC94, (1995) 199.

[5] W. Scandale; Proc. LHC Workshop (Aachen, 1990), CERN 90$10,760$.

[6] S.P. Møller et al.; Nucl. Instr. Meth. B84 (1994) 434.

[7] V.M. Biryukov; Nucl. Instr. Meth. B53 (1991) 202.

[8] A.M. Taratin et al.; Nucl. Instr. Meth. B58 (1991) 103.

[9] S. Bardin; CERN SL/Note 92-52 (AP).

[10] A. Asseev et al.; Nucl. Instr. Meth. A324 (1993) 31.

[11] S. Weisz and the RD22 collaboration; Proc. 1993 IEEE Particle Accelerator Conference, 26.

[12] E.M. Hansen; Private Communication

[13] W. Herr; CERN SL/92-53 (AP).

[14] G. Vuagnin; CERN SL/Note 95-24 (AP).

[15] K. Elsener et al.; SL-MD Note 170 (1995). 